\title{
Autonomous Heat Leakage Detection from Unmanned Aerial Vehicle-Mounted Thermal Cameras
}

\author{
Burak Kakillioglu, Senem Velipasalar and Tarek Rakha \\ Syracuse University \\ Syracuse, NY, United States \\ [bkakilli,svelipas,trakha]@syr.edu
}

\begin{abstract}
The efficiency of thermal insulation of buildings has direct impact on power consumption for heating and ventilation. Thermal leaks are critical defects on building insulation. Infrared sensitive handheld thermal cameras are commonly used to perform manual inspection of buildings to detect thermal leaks by human experts. In this paper, we propose an algorithm that autonomously detects heat leakages from thermal images of building structures. The target platform for the proposed algorithm is an unmanned aerial vehicle (UAV) that collects thermal images of buildings, and detects heat leakages autonomously. Using UAVs, as opposed to the current practice of doing manual inspection, not only speeds up the process, but also provides the capability of capturing data from hard to reach places, such as roofs, in a more convenient and safe manner. For evaluation, thermal images have been captured both from inside, by a handheld camera, and outside. The outside thermal images have been captured by either a handheld camera or an IR camera installed on a UAV. Experimental results show that the proposed approach is very promising with overall precision and recall rates of $91 \%$ and $89 \%$, respectively on handheld dataset, and $34 \%$ and $77 \%$, respectively on the UAV dataset.
\end{abstract}

\section{CCS CONCEPTS}

- Computer systems organization $\rightarrow$ Robotic autonomy; Embedded software;

\section{KEYWORDS}

Heat leakage, thermal leak, detection, UAV inspection

\section{ACM Reference Format:}

Burak Kakillioglu, Senem Velipasalar and Tarek Rakha. 2018. Autonomous Heat Leakage Detection from Unmanned Aerial Vehicle-Mounted Thermal Cameras. In International Conference on Distributed Smart Cameras (ICDSC '18), September 3-4, 2018, Eindhoven, Netherlands. ACM, New York, NY, USA, 6 pages. https://doi.org/10.1145/3243394.3243696

This work has been funded in part by National Science Foundation (NSF) under Grant 1739748.

Permission to make digital or hard copies of all or part of this work for personal or classroom use is granted without fee provided that copies are not made or distributed for profit or commercial advantage and that copies bear this notice and the full citation on the first page. Copyrights for components of this work owned by others than ACM must be honored. Abstracting with credit is permitted. To copy otherwise, or republish, to post on servers or to redistribute to lists, requires prior specific permission and/or a fee. Request permissions from permissions@acm.org.

ICDSC '18, September 3-4, 2018, Eindhoven, Netherlands

(C) 2018 Association for Computing Machinery.

ACM ISBN 978-1-4503-6511-6/18/09...\$15.00

https://doi.org/10.1145/3243394.3243696

\section{INTRODUCTION}

Achieving substantial energy savings through existing building upgrades is a result of accurate and reliable energy audits, followed by retrofitting strategies that respond accordingly. However, reviews of actual savings in existing building retrofits show a wide discrepancy between predicted and delivered savings. Imprecise energy audits can result in either (i) lower than expected energy savings, (ii) no energy savings or (iii) occasional increase in energy use. This leads to worse environmental impacts, waste of investment capital, and a decrease in the rate of adoption for energy efficiency retrofits. This is typically a result of many challenges that energy auditors face, including insufficient building information that leads to misrepresentation in energy models, overestimated savings, ineffective selection of improvement strategies and incomprehensive improvement scope that results in missed improvement opportunities [12]. For example, in large commercial buildings, energy auditors focus on exciting technical challenges such as demand-control-ventilation or integration of solar energy, whereas building envelope issues such as window, air sealing and insulation are neglected [13]. Current methods of inspecting building envelopes are time consuming, costly or have low accuracy. Qualitative methods include moisture tests and fan pressurization tests, which are used to identify inadequacies in building shells, but are limited in terms of comprehensive thermal performance evaluation [4]. A quick, accurate and quantitative method for non-destructive testing of structures is infrared thermography, which makes detecting anomalies in building construction possible and reliable [2].

Aerial building inspection has gained significant interest over the last decade with the emerging UAV technology. Various scenarios have been addressed including aerial inspection of heat losses, and solution strategies have been studied in the literature. Eschmann et al. [5] used a UAV to capture high resolution and frequency images of a building from facade, and performed sub-millimeter range crack inspections after reconstructing single very high resolution building image. Maurellio et al. [9] used a UAV with RGB camera and a hand-held IR camera for 3D thermal profiling. They have performed 3D reconstruction with SfM (structure from motion) on RGB images captured in series and then mapped corresponding thermal information to the overlay. Mavromatidis et al. [10] proposed the use of impulse thermography captured by UAV. They highlighted that UAV-thermography can be implemented and it delivers the advantage of reaching inaccessible areas of a structure. Martinez et al. [8] used a thermal camera mounted on a UAV to inspect heat losses through the windows. Authors put a fixed $7^{\circ} \mathrm{C}$ threshold to differentiate the thermal leaks and find a region of interest (ROI). They then further analyze the ROI to check if the frame of the windows also has the heat leakage problem by classifying 


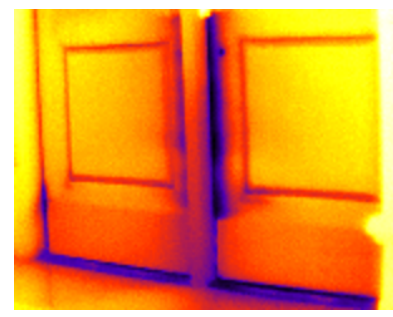

(a) Thermal image

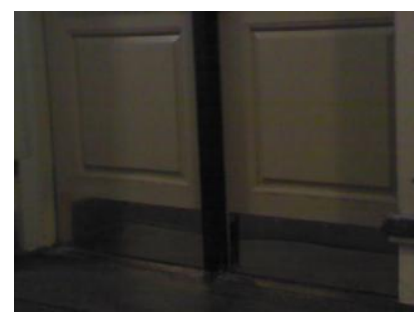

(b) RGB Correspondence
Figure 1: Example of a significant infiltration around the door frame. Cold air from outside is infiltrating through the gaps causing the edges of the door on thermal image become purple (cold is represented by purple and hot is represented by red in (a)).

the ROIs into two classes based on the uniformity of the emissivity of the material. Although this second analysis might have reduced the false positives, use of a single threshold, especially a fixed one, would not be feasible and reliable according to our experiments (see Section 4.3).

In this work, we present an inspection algorithm to autonomously detect heat leakages on building structures from aerial thermal images captured from an unmanned aerial vehicle (UAV) platform or from thermal images captured by a handheld IR camera. We have collected a set of thermal images that show heat leakages through various structures inside and outside the buildings. We have conducted a thorough experimental analysis and will discuss the effects of each parameter in the proposed algorithm.

\section{HEAT LEAKAGE DETECTION OVERVIEW}

Heat leakage is the undesired and typically uncontrolled loss or gain of energy through the building envelope. Heat leakage can occur through thermal bridging or excessive heat loss, air leakages and missing or damaged thermal insulation in the building's elements [7]. As exfiltration and infiltration occur most commonly around doors, windows, and other access points to the building, their presence is most visible on the perimeters of such access points. Thermal images of leaking doors or windows have patches of either cooler or warmer air clustered around the region, where the weather barrier is compromised [6]. Such ventilation losses can account for over half the total energy use in a building [1].

A heat leakage is usually a spot or an area which can be observed on a colored thermography as a region whose color is significantly

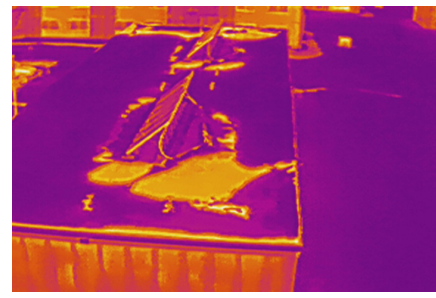

(a) Thermal image

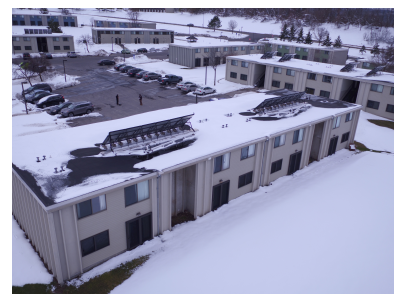

(b) RGB Correspondence
Figure 2: Snow is melted at certain parts of the roof of the building due to a thermal anomaly in the roof insulation. different than its surroundings [11]. It is observed under four different scenarios based on the season and the area where inspection is being done, i.e., inside or outside of the building. In winter season, an energy auditor seeks signs of colder regions on the thermography captured inside whereas he/she seeks hotter regions on the thermography captured outside, since the outside temperature is much lower than the inside temperature. In the summer season, the auditor does the opposite as the outside temperature is expected to be higher than inside temperature.

It is important to note that there are some instances where above definition holds for some regions on the thermal image although those regions are in fact healthy. Such cases include reflection (metal elements can heat up and down much faster than the wall itself, e.g., sign on the wall, balcony fences etc.) and shadowing.

In this work, we have captured two sets of thermal images. First, we used an FLIR ${ }^{\circledR}$ E40 handheld thermal imaging camera to manually collect a thermal image dataset. The data consists of thermal images with 160x120 resolution and corresponding RGB images with relatively higher resolution. Second, we used a UAV, more specifically a DJI ${ }^{\mathrm{TM}}$ Inspire 1 equipped with Zenmuse XT thermal camera, to autonomously capture thermal images around buildings. We processed the collected thermal images offline, but the ultimate goal is to carry out the whole processing on-board and in realtime. Figures 1 and 2 show example thermal and RGB images captured by a handheld camera inside and from a UAV-mounted camera outside, respectively. Fig. 1 has heat leakage around the door due to air infiltration. Fig. 2 shows heat leakage on the roof, since snow is melted at some parts whereas it stayed on the majority of the roof, due to an anomaly in the insulation material.

\section{PROPOSED HEAT LEAKAGE DETECTION ALGORITHM}

The proposed heat leakage detection framework is based on locating sharp and abnormal temperature variations on the thermal images. When inspecting a color-mapped thermal image, which has e.g. been taken indoors during winter season, a thermography expert identifies the leakage as a purple (cold) region surrounded by red (hot) regions as seen in Fig. 1(a). The thermal image is a 2D matrix whose cells, i.e. pixels, are temperature values. Thus, sharp intensity changes on a single-layer image can be identified by detecting edges. However, not all of the detected edges in thermal images correspond to boundaries of heat leakage regions. Purple regions in Fig. 1(a) are surely leaking parts of the door and an edge detector would separate those regions. Yet, there are other visible edges on the same image, such as the framing on the doors. Therefore, those edges must be eliminated from the edge detection output. Our proposed method successfully filters most of the false edges out by a procedure which we henceforth refer to as edge traversing, and then eventually segments out the leakage regions.

Our proposed thermal leakage detection algorithm has two main stages. The first stage is a global lookup over the thermal image to autonomously compute two threshold values. The second stage, which uses the threshold values calculated in the first stage, is a refined search along the thermal edges for determining whether an edge point belongs to the boundary of a heat leakage. The second stage also involves region growing and morphology operations for 


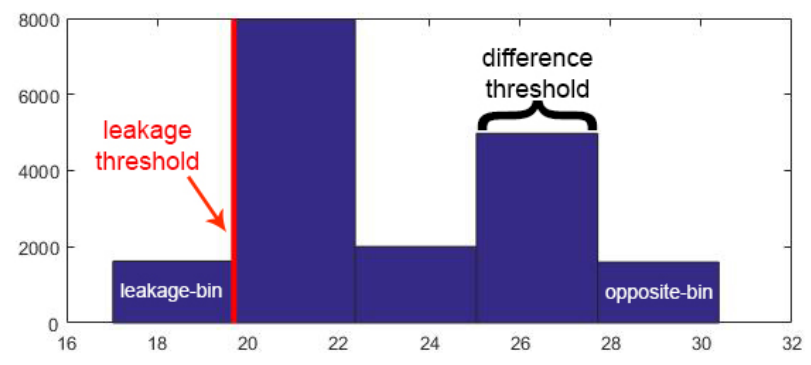

Figure 3: The difference threshold and the leakage threshold displayed on a sample histogram.

final segmentation of heat leakages. There are a few assumptions as well as some global parameters for defining a heat leakage. We will describe them and investigate and show how they could affect the results.

\subsection{Threshold Computation}

In the first stage of the algorithm, we compute two threshold values, which are referred to as the difference threshold and leakage threshold. These thresholds are used in the second stage of the algorithm when performing edge traversing as described in Sect. 3.2.

To compute these thresholds, we employ a temperature histogram, and determine the number of bins in an adaptive manner. We initially start with a 3-bin histogram. We define the leakagebin as the histogram bin where the coldest (hottest) values fall into for images whose leaks are expected to be colder (hotter) than its vicinity. We define opposite-bin as the farthest bin from the leakage-bin. We first check if the opposite-bin has enough representativeness, i.e. if there are enough pixels in it. If there are not, we remove the samples that fall into the opposite-bin, and recalculate the histogram until it has enough samples in it (more than $10 \%$ of the total number of pixels for the base settings in experiments, see Sec. 4.5).

Next, we limit the number of samples in the leakage-bin, since we assume that the number of pixels in potential leakage regions do not exceed 15\% (base setting, please see Sec. 4.5) of the total number of pixels. If the number of pixels in the leakage-bin is larger than the $15 \%$ of all pixels, we increase the number of bins of the histogram by one and recompute it. We repeat this process until the criteria is met. After the algorithm settles with an M-bin histogram, the difference threshold is set to be the width of the bin before the last, and the leakage threshold is set to be the boundary value between the leakage-bin and the bin next to it (as seen in Fig. 3).

\subsection{Segmenting Leakage Regions}

After computing the difference and leakage thresholds, region growing is performed and leakage regions are segmented. First, edges in the thermal image are extracted by using the Canny edge detector [3]. The parameters of the edge detector will be discussed in Sec. 4. As mentioned above, not all edges belong to the leakage region boundaries. Figures 5(a) and 5(b) show the original thermal image and the detected edges, respectively, and as can be seen, there are many edges that are not part of the leakage regions. To eliminate the false edges, we traverse each edge by visiting every edge pixel as depicted in Fig. 4. On the current visited pixel, we build an $\mathrm{N}$-neighborhood vector perpendicular to the edge direction. To keep an edge pixel, as part of a leakage edge, two conditions must be met. First, the maximum temperature difference in the neighborhood must be greater than the difference threshold to ensure that there is enough temperature change around the visited edge pixel. For the second condition, the extreme values in the neighborhood are compared with the leakage threshold based on the inspection type. If the leakage region is expected to be colder than its vicinity, than the smallest value in the neighborhood must be smaller than the leakage threshold. Similarly, if a leakage region is expected to be hotter, than the largest value in the neighborhood must be larger than the leakage threshold. When both of these conditions are met, the visited edge pixel and the most severe leakage pixel in the neighborhood is saved. Fig. 5(c) shows the pixels that are kept after edge traversing.

During the post processing stage, the actual leakage regions are segmented. We first generate a binary segmentation image by applying region growing to the leakage pixels that have been saved in the neighborhood of the visited edge pixels. The binary image after region growing contains the leakage region with gaps in them. To fill those gaps, morphological closing is applied as the final step. Fig. 5(d) shows the segmented heat leakage regions.

\subsection{Assumptions and Parameters}

The proposed method can differentiate cold from hot. However, in order to know which one corresponds to leakage, the algorithm needs to know the season and whether the data was collected from inside or outside. More specifically, if the season is winter, the infiltrating air will be cold. While inspecting the building from inside, the leaking parts will be colder. If the inspection is done from outside during winter, then the leaking parts will be hotter. On the other hand, the exact opposite scenario happens during the summer. Therefore, season and inspection area information are critical for heat leakage judgment, and a parameter is defined in the algorithm to indicate whether it should seek colder or hotter regions as thermal leaks. In this work, we have performed data collection and experiments during both winter and summer.

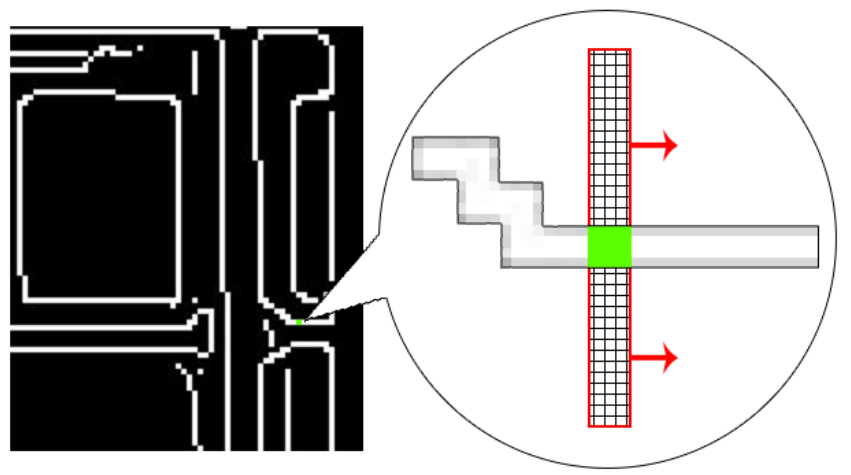

Figure 4: Edge analysis illustration. Green dot is the currently visited edge pixel. The algorithm moves along the edge direction and checks $\mathbf{N}$-neighborhood (grid bar with length $\mathrm{N}$-pixel) that is perpendicular to edge direction. If $\mathrm{min}<$ leakage threshold for cold leaks (or max > leakage threshold for hot leaks) and (max-min) $>$ difference threshold, the edge pixel is kept as leakage edge. 


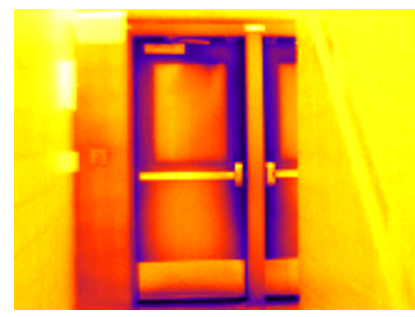

(a) Thermal image

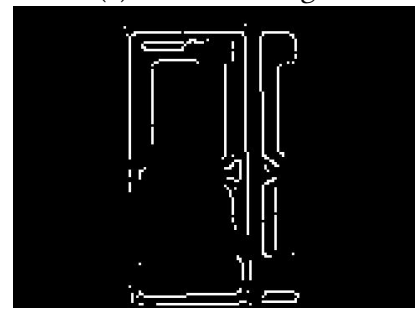

(c) Processed edge image

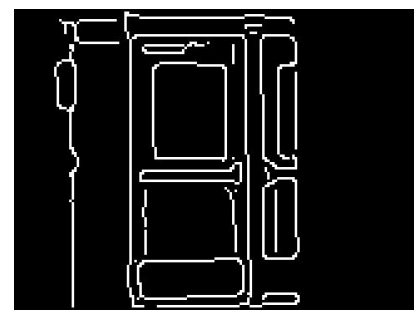

(b) Thermal edges

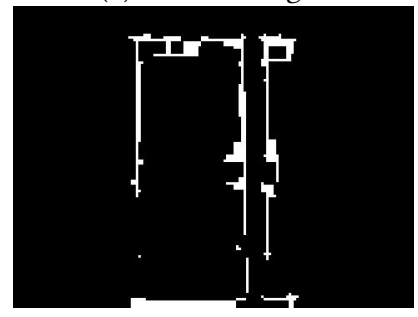

(d) Post processing
Figure 5: (a) Thermal image, (b) detected edges, (c) output after edge following/traversing, (d) output of leakage detection.

As described above, in the first stage of the algorithm, we set criteria for representativeness of leakage-bin and opposite-bin. It is assumed that the leakage region is smaller than $15 \%$ of the image. Also, the number of samples in the opposite-bin needs to be larger than $10 \%$ of the total number of pixels to be representative enough These ratios have been set empirically, and their effect on results is discussed in Sec. 4.

For edge detection, the standard deviation of the smoothing Gaussian, and the low and high threshold values have been set to be $\sigma=\sqrt{2}, 0.08$ and 0.2 , respectively. We have chosen small values for thresholds, since we want as many edges to be preserved as possible. Although this may introduce many false edges, they are eliminated at the subsequent stages. In addition, reducing the number of misses is more critical than having false positives for this application. The only parameter used at the edge following procedure is the neighborhood size. An empirically defined number 13 is used as the neighborhood size in the base settings in our experiments. To determine the neighborhood size, one should consider the resolution of the image. Increasing the neighborhood size too much would introduce more false positive detections.

In the region growing part of the post processing, we have used $0.1^{\circ} \mathrm{C}$ as the maximum allowable temperature difference to merge the neighboring pixels with the leakage region. Finally, for morphological closing operation, we have used a disk shaped structuring element with radius 3 .

\section{EXPERIMENTAL ANALYSIS}

\subsection{Data Collection}

In this work, we have collected two different datasets. First dataset was collected by using a handheld thermal imaging camera, which outputs $160 \times 120$ thermal images. Images have been taken from both inside and outside of buildings, from different distances and viewing angles. The second dataset was collected by a UAV-mounted thermal camera covering the whole envelope of the buildings. A total of 100

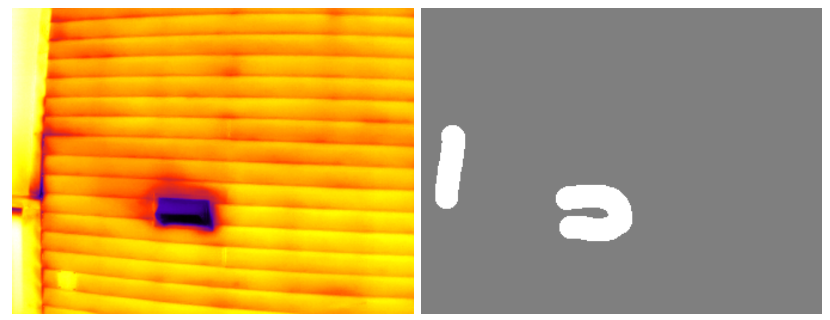

Figure 6: Aerial thermal image and the marked ground truth leak region (in white).

thermal images which have resolution of 256x336 were labeled and used in the experiments.

\subsection{Performance Assessment}

Unlike object detection and segmentation from RGB images, it is not very straightforward to obtain ground truth information for thermal leakage regions on IR images. One reason is that they do not appear in a certain pattern or contour. They can emerge as random silhouettes. Another reason is that identification of them requires thermography expertise. We have performed two different analyses for our two datasets (handheld camera and the UAV datasets).

The evaluation of the algorithm on the handheld camera dataset has been done by our thermography expert. To remove the bias from his assessment, he is first asked to diagnose the leakage regions on the thermal images, and then identify the matching or missed detections in the algorithm's output.

On the other hand, the evaluation on the UAV dataset has been done by using pixel-wise annotated ground truth for each image. Thermography experts were asked to provide segmentations of thermal leaks on every image. Exact pixel-wise segmentation can be highly subjective and the precise shape of the leakage region is not essential for locating anomaly regions. Thus, experts were asked to roughly mark the leakage regions by using a thick masking brush tool (see Fig. 6). To define correct detection of leakage, we use the ratio $R=\frac{A_{D \cap G}}{A_{D}}$, where $A_{D}$ is the number of pixels in the detection output and $A_{D \cap G}$ is the number of pixels in the intersection of the detection output with the marked ground truth region. $R$ is calculated for every detection region. If $R>0.1$, it is considered as correct detection. If $R<0.1$, it is considered as a false positive. If no detection region intersects with a ground truth region, it is considered as a miss or a false negative. If there is more than one match with a ground truth region, only one detection is counted.

\subsection{Unreliable Approach: Using Fixed Threshold}

As briefly discussed above, although it is fairly straightforward, using a fixed threshold value for leakage segmentation would not work, due to following reasons. First, every thermal leak has different severity levels. Second, thermal leaks at different parts of the building may exist due to similar deficiencies, but not all of them could be diagnosed by the same threshold value. Fig. 7 shows outputs of using different but fixed threshold values for two indoor images. As can be seen, the threshold value of $10^{\circ} \mathrm{C}$ works well for the first image, whereas nothing is detected in the second image. When we increase the threshold value to $15^{\circ} \mathrm{C}$, the detection for the second image looks fine. However, amount of false detection 


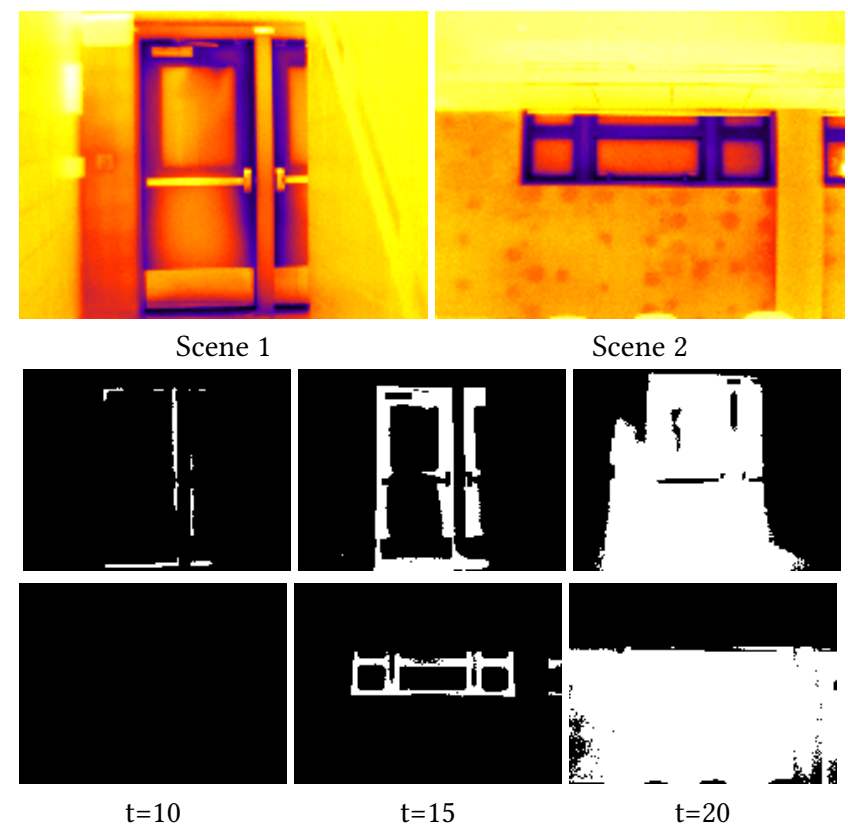

Figure 7: Fixed thresholding is unreliable because it cannot be generalized to be used for every image. First row is the thermal images. Three columns of second and third rows are detection outputs of simple thresholding with threshold values $10^{\circ} \mathrm{C}, 15^{\circ} \mathrm{C}, 20^{\circ} \mathrm{C}$, from left to the right.

increases in the first image. Thus, a fixed threshold is not reliable to be used on every capture, and it must be set dynamically per image as proposed in our method.

\subsection{Hand-held Camera Capture Results}

With this dataset, we have tested our approach on 113 thermal images that contain a total of 326 heat leakage regions. Our algorithm successfully detects 291 of the 326 leakage regions, and reports 28 extra regions, which are false positives. Thus, the precision and recall rates are $91 \%$ and $89 \%$, respectively.

Fig. 8 shows example thermal images with their corresponding heat leakage detection results. The third column of this image presents an especially challenging scenario, namely a thermal bridge. Here, the leakage is not around a window or door, but is due to the nails on the studs in the wall. The proposed approach can successfully detect the heat leakage in this scenario as well.

\subsection{UAV Capture Results}

As mentioned above, this dataset was captured by a UAV-mounted camera, while UAV was flown around the buildings. Ground truth for 100 thermal images were obtained from thermography experts and those images have been used for evaluation. Fig. 9 shows detection results for four example images together with the original thermal image and corresponding ground truth labeling.

Table 1 shows results under different parameter settings. Base settings are the following. Canny edge detector parameters: Low threshold is 0.04 , high threshold is 0.2 , and sigma is $\sqrt{2}$. Minimum representativeness for opposite-bin is $10 \%$ and maximum representativeness for leakage-bin is $15 \%$. Neighborhood size is 13 pixels.

\begin{tabular}{l|ccccc} 
& TP & FP & FN & Precision & Recall \\
\hline Base Settings & 163 & 320 & 52 & 0.337 & 0.758 \\
Canny sigma=1 & 165 & 333 & 50 & 0.331 & 0.767 \\
Canny sigma=2 & 163 & 311 & 52 & 0.344 & 0.758 \\
Canny th=[0.04 0.1] & 163 & 320 & 52 & 0.337 & 0.758 \\
Canny th=[0.16 0.4] & 160 & 305 & 55 & 0.344 & 0.744 \\
O.B. Repr $=0.2$ & 160 & 299 & 55 & 0.349 & 0.744 \\
O.B. Repr=0.05 & 160 & 314 & 55 & 0.338 & 0.744 \\
L.B. Repr=0.2 & 165 & 327 & 50 & 0.335 & 0.767 \\
L.B. Repr=0.3 & 169 & 397 & 46 & 0.299 & 0.786 \\
Neigborhood=9 & 157 & 335 & 58 & 0.319 & 0.730 \\
Neigborhood=11 & 165 & 340 & 50 & 0.327 & 0.767 \\
Neigborhood=15 & 163 & 289 & 52 & 0.361 & 0.758 \\
Min det. size $=5$ & 152 & 251 & 63 & 0.377 & 0.707 \\
Min det. size=10 & 144 & 213 & 71 & 0.403 & 0.670 \\
Min det. size=20 & 130 & 179 & 85 & 0.421 & 0.605
\end{tabular}

Table 1: Effects of different parameter values. (see Sec. 4.5) O.B. and L.B. are abbreviations for opposite-bin and leakagebin respectively.

Every row in Table 1 is the result of an experiment in which the effect of only one parameter is analyzed while the other parameters are set to the base values stated above. The last three rows in Table 1 show the effect of discarding very small detected leakage regions. As can be seen, this reduces the number of false positives, and increases the precision with the tradeoff of reducing the number of correct detections.

\section{DISCUSSION AND FUTURE WORK}

Overall, the proposed approach provides promising performance across datasets covering various scenarios, such as different capture methods and different seasons. The performance is especially good for the handheld camera dataset, with precision and recall rates of $91 \%$ and $89 \%$, respectively. The performance on handheld camera dataset is better than the UAV dataset, and this is mostly due to the process of ground truth labeling and evaluation. In other words, the handheld camera results have been individually inspected by an expert, whereas the UAV dataset results have been evaluated by computer by using the ground truth labeling. For the former, the thermography expert identified problematic and unreliable thermal images containing reflections and shadows etc. and did not take unreliable images into consideration. For the latter, these cases caused increased number of false positives because evaluation algorithm cannot differentiate a reflection problem from actual leaks. Furthermore, we can say that the labeling has multiple possible interpretations due to the context of materials and leakage. In other words, while one expert might consider the change in materials as a possible source of leakage, others might consider that the depth of the material is a variable and perhaps that what is seen is not a leakage and more of a specular reflection. The consistency of the algorithm outcomes matches the inconsistency of possible interpretations. However, in cases where there are no material differences, the accuracy is expected to rise.

Moreover, the number of missed detections can be reduced with further parameter tuning. Although this might increase the number of false positives, the application of building envelope inspection is more tolerant to having false positives compared to missing actual leaks. 

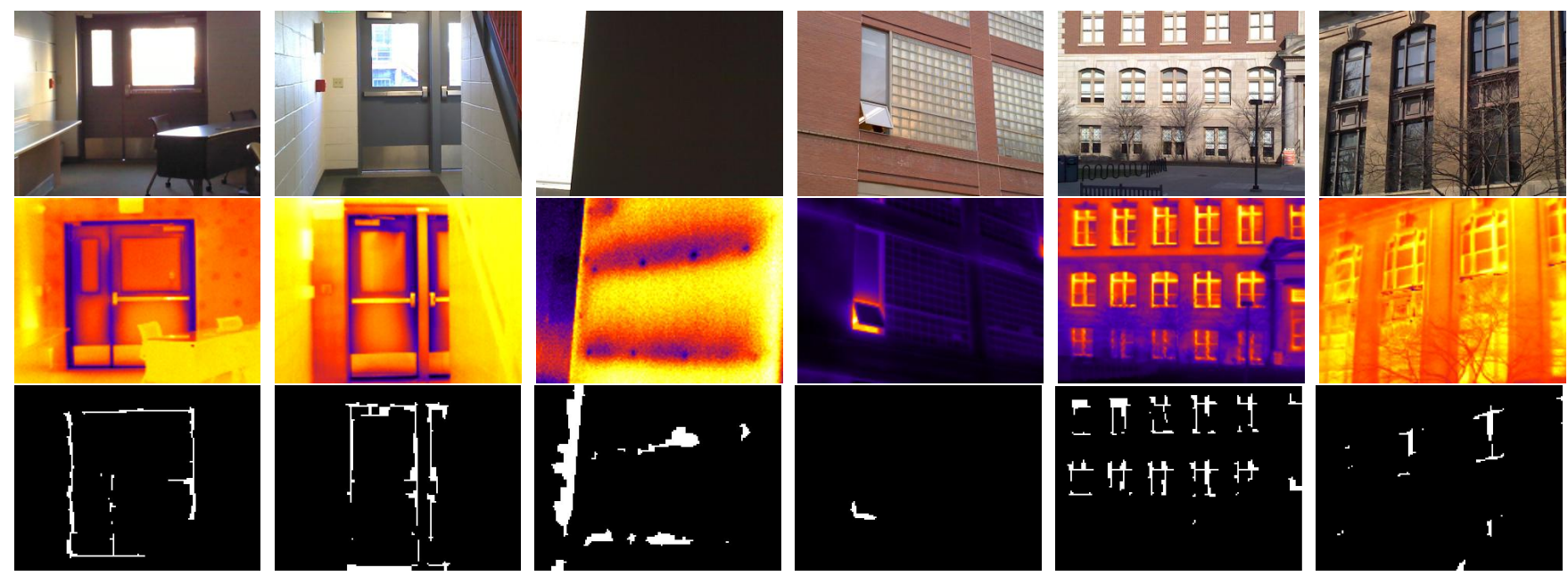

Figure 8: Experimental results from different distances, different agles, and various sides of buildings. In each 12 triplet, the top images are the RGB correspondances of the scenes, middle ones are captured thermal images, and the bottom ones are the segmented leakage regions.

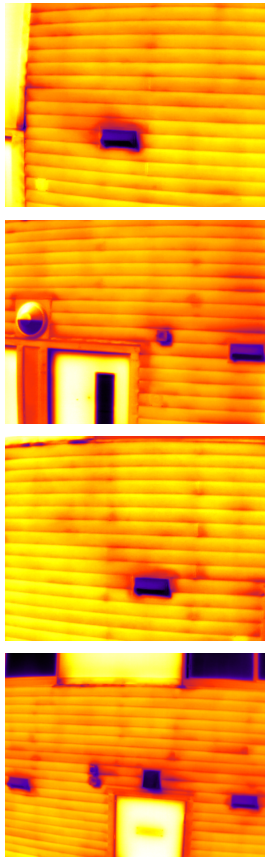

Thermal Images
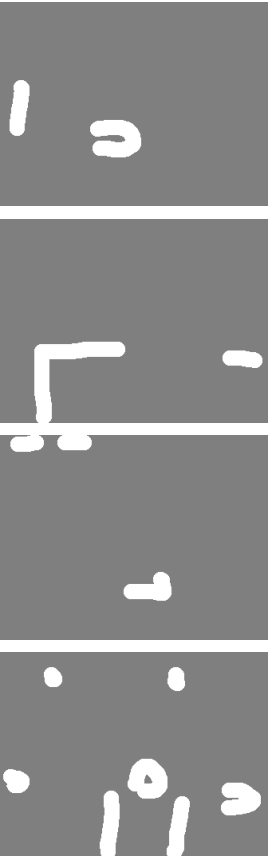

Ground Truths
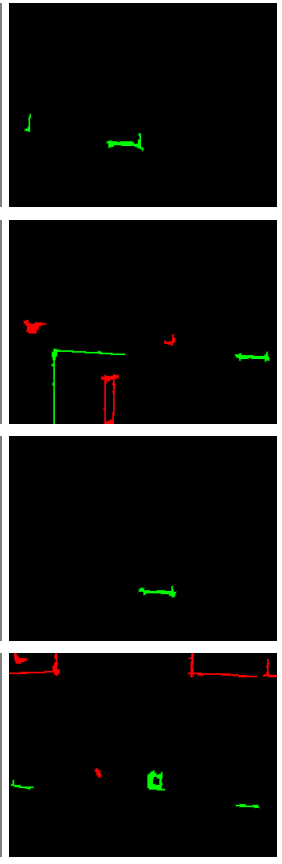

Detections
Figure 9: Results on UAV-captured data. Green and red color denote true positives and false positives, respectively.

Future work includes addressing the aforementioned reflection and shadow issues so that the algorithm can automatically eliminate those instances. In addition, incorporation of semantics would enable the algorithm to perform the analysis based on the structure type (such as roof versus facade) or context.

\section{CONCLUSION}

An algorithm has been presented for autonomous detection of heat leakages from thermal images. The goal is to perform fast, reliable and safe inspection of buildings to detect thermal infiltration/exfiltration problems by using UAVs and UAV-mounted thermal cameras. The proposed autonomous heat leakage segmentation framework analyzes thermal images independent of the type of the inspected structure and the temperature distribution of the

\section{REFERENCES}

[1] John Armstrong, Ken Butcher, and Justin Rowe. 2003. CIBSE concise handbook. Chartered Institution of Building Services Engineers.

[2] Eva Barreira and Vasco P de Freitas. 2007. Evaluation of building materials using infrared thermography. Construction and Building Materials 21, 1 (2007), 218-224.

[3] John Canny. 1987. A computational approach to edge detection. In Readings in Computer Vision. Elsevier, 184-203.

[4] Itai Danielski and Morgan Fröling. 2015. Diagnosis of BuildingsâĂŹ Thermal Performance-A Quantitative Method Using Thermography Under Non-steady State Heat Flow. Energy Procedia 83 (2015), 320-329.

[5] C Eschmann, CM Kuo, CH Kuo, and C Boller. 2012. Unmanned aircraft systems for remote building inspection and monitoring. In 6th European workshop on structural health monitoring. 1-8.

[6] Matthew Fox, David Coley, Steve Goodhew, and Pieter De Wilde. 2014. Thermography methodologies for detecting energy related building defects. Renewable and Sustainable Energy Reviews 40 (2014), 296-310.

[7] Angeliki Kylili, Paris A Fokaides, Petros Christou, and Soteris A Kalogirou. 2014. Infrared thermography (IRT) applications for building diagnostics: A review. Applied Energy 134 (2014), 531-549.

[8] JR Martinez-De Dios and Anibal Ollero. 2006. Automatic detection of windows thermal heat losses in buildings using UAVs. In Automation Congress, 2006. WAC'06. World. IEEE, 1-6.

[9] Matthew Louis Mauriello and Jon E Froehlich. 2014. Towards automated thermal profiling of buildings at scale using unmanned aerial vehicles and 3Dreconstruction. In Proceedings of the 2014 ACM International Foint Conference on Pervasive and Ubiquitous Computing: Adjunct Publication. ACM, 119-122.

[10] LE Mavromatidis, JL Dauvergne, R Saleri, and JC Batsale. 2014. First experiments for the diagnosis and thermophysical sampling using impulse IR thermography from Unmanned Aerial Vehicle (UAV). In Qirt conference.

[11] Tarek Rakha, Amanda Liberty, Alice Gorodetsky, Burak Kakillioglu, and Senem Velipasalar. 2018. Heat Mapping Drones: An Autonomous Computer-VisionBased Procedure for Building Envelope Inspection Using Unmanned Aerial Systems (UAS). Technology| Architecture+ Design 2, 1 (2018), 30-44.

[12] Ian Shapiro. 2011. 10 Common problems in energy audits. ASHRAE fournal 53, 2 (2011), 26.

[13] P.E. Shapiro, Ian. 2009. Energy Audits In Large Commercial Office Buildings. ASHRAE fournal 51, 1 (01 2009), 18-27. https://search-proquest-com.libezproxy2. syr.edu/docview $/ 220459268$ ?accountid=14214 environment. 